\title{
BLOCKS WITH SMALL-DIMENSIONAL BASIC ALGEBRA BENJAMIN SAMBALED
}

(Received 26 May 2020; accepted 23 June 2020; first published online 21 September 2020)

\begin{abstract}
Linckelmann and Murphy have classified the Morita equivalence classes of $p$-blocks of finite groups whose basic algebra has dimension at most 12. We extend their classification to dimension 13 and 14 . As predicted by Donovan's conjecture, we obtain only finitely many such Morita equivalence classes.
\end{abstract}

2020 Mathematics subject classification: primary 20C05; secondary 16D90.

Keywords and phrases: basic block algebra, Morita equivalence, Donovan's conjecture.

\section{Introduction}

Let $F$ be an algebraically closed field of characteristic $p>0$. Donovan's conjecture (over $F$ ) states that for every finite $p$-group $D$ there are only finitely many Morita equivalence classes of $p$-blocks of finite groups with defect group $D$. Since a general proof seems elusive at present, mathematicians have focused on certain families of $p$-groups $D$. This has culminated in a proof of Donovan's conjecture for all abelian 2-groups by Eaton and Livesey [4]. A different approach, introduced by Linckelmann [11], aims to classify blocks $B$ with a given basic algebra $A$. Recall that $A$ is the unique $F$-algebra (up to isomorphism) of smallest dimension which is Morita equivalent to $B$. Linckelmann and Murphy $[11,12]$ have classified all blocks $B$ such that $\operatorname{dim} A \leq 12$. Since the order of a defect group is bounded in terms of $\operatorname{dim} A$ (see next section), one expects only finitely many such blocks up to Morita equivalence. Indeed, the list in [11] is finite. We extend their classification as follows.

THEOREM 1.1. Let $B$ be a block of a finite group with basic algebra $A$.

(I) If $\operatorname{dim} A=13$, then $B$ is Morita equivalent to one of the following block algebras:

(a) $F C_{13}(p=13)$;

(b) the principal 13-block of $\operatorname{PSL}(3,3)$ with defect 1 ;

This work is supported by the German Research Foundation (SA 2864/1-2 and SA 2864/3-1).

(C) 2020 Australian Mathematical Publishing Association Inc. This is an Open Access article, distributed under the terms of the Creative Commons Attribution-NonCommercial-NoDerivatives licence (http://creativecommons.org/licenses/by-nc-nd/4.0/), which permits non-commercial re-use, distribution, and reproduction in any medium, provided the original work is unaltered and is properly cited. The written permission of Cambridge University Press must be obtained for commercial re-use or in order to create a derivative work. 
(c) the principal 17-block of $\operatorname{PSL}(2,16)$ with defect 1 ;

(d) the principal 2-block of $\operatorname{PGL}(2,7)$ with defect group $D_{16}$;

(e) a nonprincipal 2-block of 3.M $M_{10}$ with defect group $S D_{16}$;

(f) a nonprincipal 7-block of $6 . A_{7}$ with defect 1 .

(II) If $\operatorname{dim} A=14$, then $B$ is Morita equivalent to one of the following block algebras:

(a) $F D_{14}(p=7)$;

(b) the principal 5-block of $S_{5}$ with defect 1 ;

(c) the principal 7-block of $\mathrm{PSU}(3,3)$ with defect 1 ;

(d) the principal 19-block of $\operatorname{PSL}(2,37)$ with defect 1 .

The bulk of the proof is devoted to the nonexistence of a certain block with extraspecial defect group of order 27 and exponent 3 . The methods are quite different from those in [12]. For some of the Brauer tree algebras occurring in [11] no concrete block algebra was given. For future reference we provide explicit examples in Table 1 . Here, $B_{0}$ and $B_{1}$ denote the principal block and a suitable nonprincipal block, respectively.

TABLE 1. Morita classes.

\begin{tabular}{ccc}
\hline $\operatorname{dim}(A)$ & $D$ & Morita classes \\
\hline$\leq 5$ & $|D|=\operatorname{dim}(A)$ & $F D$ \\
6 & $C_{3}$ & $F S_{3}$ \\
7 & $C_{5}$ & $B_{0}\left(A_{5}\right)$ \\
& $C_{7}$ & $F C_{7}$ \\
8 & $C_{7}$ & $B_{0}(\mathrm{PSL}(2,13))$ \\
& $|D|=8$ & $F D$ \\
9 & $C_{9}$ & $F C_{9}, B_{0}(\mathrm{PSL}(2,8))$ \\
& $C_{3} \times C_{3}$ & $F\left[C_{3} \times C_{3}\right], B_{1}\left(2 .\left(S_{3} \times S_{3}\right)\right)$ \\
10 & $C_{5}$ & $F D_{10}$ \\
& $C_{11}$ & $B_{0}(\mathrm{PSL}(2,32))$ \\
11 & $C_{7}$ & $B_{0}(\mathrm{PSL}(2,7))$ \\
& $D_{8}$ & $F S_{4}$ \\
& $C_{11}$ & $F C_{11}$ \\
& $C_{13}$ & $B_{0}(\mathrm{PSL}(2,25))$ \\
12 & $C_{2} \times C_{2}$ & $F A_{4}$ \\
13 & $C_{7}$ & $B_{1}\left(6 . A_{7}\right)$ \\
& $C_{13}$ & $B_{0}(\mathrm{PGL}(2,7))$ \\
& $D_{16}$ & $B_{1}\left(3 . M_{10}\right)$ \\
& $S D_{16}$ & $B_{0}(\mathrm{PSL}(2,16))$ \\
& $C_{17}$ & $B_{0}\left(S_{5}\right)$ \\
& $C_{5}$ & $F D_{14}, B_{0}(\mathrm{PSU}(3,3))$ \\
& $C_{7}$ & $B_{0}(\mathrm{PSL}(2,37))$ \\
\hline & $C_{19}$ &
\end{tabular}


For basic algebras of dimension 15 there are still only finitely many corresponding Morita equivalence classes of blocks, but we do not know if a certain Brauer tree algebra actually occurs as a block. The details are described in Section 5.

\section{Preliminaries}

Before we start the proof of Theorem 1.1, we introduce a number of tools, some of which were already applied in [11]. (For more detailed definitions, see [17].)

Probably the most important Morita invariant of a block $B$ is the Cartan matrix $C$. This is a nonnegative, integral, symmetric, positive definite and indecomposable matrix of size $l(B) \times l(B)$ where $l(B)$ denotes the number of simple modules of $B$. Since the simple modules of a basic algebra are one-dimensional, the sum of the entries of $C$ equals $\operatorname{dim} A$ in the situation of Theorem 1.1. The largest elementary divisor of $C$ is the order of a defect group $D$ of $B$ and therefore a power of $p$. In particular, $|D|$ is bounded in terms of $\operatorname{dim} A$. Another Morita invariant is the isomorphism type of the centre $\mathrm{Z}(B)$ of $B$. In particular, in the situation of Theorem 1.1,

$$
k(B):=\operatorname{dim} Z(B)=\operatorname{dim} Z(A) \leq \operatorname{dim} A .
$$

Since we encounter many blocks of defect 1 in the sequel, we construct them first.

Proposition 2.1. Suppose that B is a p-block of a finite group with defect 1 and basic algebra $A$. Then $m:=(p-1) / l(B)$ is an integer, called the multiplicity of $B$. If $l(B)=1$, then $\operatorname{dim} A=p$. If $l(B)=2$, then $\operatorname{dim} A \in\{2 p, m+5\}$. If $l(B)=3$, then $\operatorname{dim} A \in\{3 p, m+9, m+11,4 m+6\}$. Moreover, if $\operatorname{dim} A \in\{13,14\}$, then only the blocks in Theorem 1.1 occur up to Morita equivalence.

Proof. By the Brauer-Dade theory, $B$ is determined up to Morita equivalence by a planarly embedded Brauer tree, the multiplicity $m$ and the position of the so-called exceptional vertex if $m>1$. For precise definitions we refer to [14, Chapter 11]. If $l(B)=1$, then $B$ has Cartan matrix $(p)$ and the result follows (the Brauer tree has only two vertices). We now construct the Brauer trees and Cartan matrices for $l(B) \in\{2,3\}$. The exceptional vertex is depicted by a black dot (if $m>1$ ).

(i)

$$
\longrightarrow \quad C=\left(\begin{array}{cc}
m+1 & m \\
m & m+1
\end{array}\right) \quad \operatorname{dim} A=4 m+2=2 p
$$

This case occurs for $B=F D_{2 p}=A$. If $p=7$, we get $\operatorname{dim} A=14$.

$$
\longrightarrow \quad C=\left(\begin{array}{cc}
m+1 & 1 \\
1 & 2
\end{array}\right) \quad \operatorname{dim} A=m+5=\frac{p+9}{2}
$$

This case occurs for the principal block of $\operatorname{PSL}(2, q)$ whenever $p$ divides $q+1$ exactly once (see [2, Section 8.4.3]). By Dirichlet's theorem there always exists a prime $q \equiv-1+p\left(\bmod p^{2}\right)$ which does the job. Choosing $(p, q)$ in $\{(17,16),(19,37)\}$ yields blocks with $\operatorname{dim} A=13$ and $\operatorname{dim} A=14$, respectively. 
(iii)

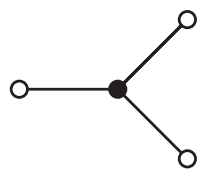

$$
C=\left(\begin{array}{ccc}
m+1 & m & m \\
m & m+1 & m \\
m & m & m+1
\end{array}\right) \quad \operatorname{dim} A=9 m+3=3 p
$$

This case occurs for $B=F\left[C_{p} \rtimes C_{3}\right]=A$. Obviously, there are no such blocks with $\operatorname{dim} A \in\{13,14\}$.

(iv)

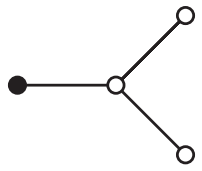

$$
C=\left(\begin{array}{ccc}
m+1 & 1 & 1 \\
1 & 2 & 1 \\
1 & 1 & 2
\end{array}\right)
$$$$
\operatorname{dim} A=m+11=\frac{p+32}{3} .
$$

We do not know if this tree always occurs as a block algebra, but it does for a nonprincipal 7-block of the 6-fold cover $6 . A_{7}$ (see [19]). This gives an example with $\operatorname{dim} A=13$. Obviously, $\operatorname{dim} A=14$ cannot occur here.

(v)

$$
\longrightarrow C=\left(\begin{array}{ccc}
m+1 & 1 & 0 \\
1 & 2 & 1 \\
0 & 1 & 2
\end{array}\right) \quad \operatorname{dim} A=m+9=\frac{p+26}{3}
$$

By [13, Proposition 2.1], there exists a prime $q$ such that $p$ divides $q^{3}-1$ exactly once. Then the principal block of $\operatorname{GL}(3, q)$ has this form by Fong and Srinivasan [6]. The principal 13-block of $\operatorname{PSL}(3,3)$ is an example with $\operatorname{dim} A=13$. Again, $\operatorname{dim} A=14$ is impossible here.

(vi)

$$
\longrightarrow C=\left(\begin{array}{ccc}
m+1 & m & 0 \\
m & m+1 & 1 \\
0 & 1 & 2
\end{array}\right) \quad \operatorname{dim} A=4 m+6=\frac{4 p+14}{3}
$$

Again by [13, Theorem 1], there exists a prime $q$ such that the principal block of $\mathrm{GU}(3, q)$ has this form. The principal 7-block of PSU(3,3) is an example with $\operatorname{dim} A=14$. On the other hand, $\operatorname{dim} A=13$ cannot occur.

Finally, if $l(B) \geq 4$, then the trace of $C$ is greater than or equal to 8 and we need at least six positive off-diagonal entries to ensure that $C$ is symmetric and indecomposable. Hence, $\operatorname{dim} A \leq 14$ can only occur if $l(B)=4, \operatorname{dim} A=14, m=1$ and the Brauer tree is a line. This happens for the principal 5-block of $S_{5}$.

In order to investigate blocks of larger defect, we develop some more advanced methods. The decomposition matrix $Q=Q_{1}$ of $B$ is nonnegative, integral and 
indecomposable of size $k(B) \times l(B)$ such that $Q^{t} Q=C$. Given $\operatorname{dim} A$, there are only finitely many choices for $Q$. Richard Brauer introduced the so-called contribution matrix

$$
M=M^{1}:=|D| Q C^{-1} Q^{\mathrm{t}} \in \mathbb{Z}^{k(B) \times k(B)} .
$$

The heights of the irreducible characters of $B$ are encoded in the $p$-adic valuation of $M$ (see [17, Proposition 1.36]). As usual, we denote the number of irreducible characters of $B$ of height $h \geq 0$ by $k_{h}(B)$. If $k_{0}(B)<k(B)$, then $D$ is nonabelian according to Kessar and Malle's [10] solution of one half of Brauer's height-zero conjecture.

The 2-blocks occurring in Theorem 1.1 are determined by the next proposition.

Proposition 2.2. Let $B$ be a block of a finite group with Cartan matrix $C=\left(\begin{array}{ll}5 & 2 \\ 2 & 4\end{array}\right)$. Then $B$ is Morita equivalent to the principal 2-block of $\operatorname{PGL}(2,7)$ or to a nonprincipal block of 3.M. Moreover, there is no block with Cartan matrix

$$
\left(\begin{array}{lll}
5 & 1 & 1 \\
1 & 2 & 0 \\
1 & 0 & 2
\end{array}\right) \text { or }\left(\begin{array}{lll}
6 & 1 & 0 \\
1 & 2 & 1 \\
0 & 1 & 2
\end{array}\right) \text {. }
$$

PROOF. All three matrices have largest elementary divisor 16 . Therefore, $p=2$ and a defect group $D$ of $B$ has order 16. For the first matrix, the possible decomposition matrices are

$$
\left(\begin{array}{ll}
1 & 1 \\
1 & 1 \\
\cdot & 1 \\
\cdot & 1 \\
1 & . \\
1 & . \\
1 & .
\end{array}\right), \quad\left(\begin{array}{ll}
2 & 1 \\
\cdot & 1 \\
\cdot & 1 \\
\cdot & 1 \\
1 & .
\end{array}\right) .
$$

The diagonal of the contribution matrix $M^{1}$ is $(5,5,5,5,4,4,4)$ or $(13,5,5,5,4)$. It follows that $k_{0}(B)=4$ (the first four characters have height 0 ). By [17, Theorem 13.6], the Alperin-McKay conjecture holds for all 2-blocks of defect 4. Thus, $k_{0}\left(B_{D}\right)=4$ where $B_{D}$ is the Brauer correspondent of $B$ in $\mathrm{N}_{G}(D)$. Now $B_{D}$ dominates a block $\overline{B_{D}}$ of $\mathrm{N}_{G}(D) / D^{\prime}$ with abelian defect group $D / D^{\prime}$. By [14, Theorem 9.23],

$$
k\left(\overline{B_{D}}\right)=k_{0}\left(\overline{B_{D}}\right) \leq k_{0}\left(B_{D}\right)=4 .
$$

Now [17, Proposition 1.31] implies $\left|D / D^{\prime}\right|=4$. Hence, $D$ is a dihedral group, a semidihedral group or a quaternion group. A look at [17, Theorem 8.1] (the Cartan matrices in (5a) and (5b) are mixed up), tells us that $k(B)=7$ and $D \in\left\{D_{16}, S D_{16}\right\}$. The corresponding Morita equivalence classes were computed by Erdmann [5] (see [9, Appendix] for a definitive list). Only the two stated examples occur up to Morita equivalence.

For the second matrix there is only one possible decomposition matrix and we obtain similarly that $k_{0}(B)=4$ and $k(B)=7$. By [17, Theorem 8.1], $D \cong D_{16}$. However, it can be seen from [9, Appendix] that there are no such blocks (all Cartan invariants 
are positive). Nevertheless, $C$ occurs as Cartan matrix with respect to a suitable basic set (for the principal block of $\operatorname{PSL}(2,17)$, for instance).

In the last case there are two feasible decomposition matrices:

$$
\left(\begin{array}{ccc}
2 & \cdot & \cdot \\
1 & 1 & . \\
1 & \cdot & . \\
\cdot & 1 & 1 \\
. & \cdot & 1
\end{array}\right),\left(\begin{array}{lll}
1 & 1 & \cdot \\
1 & \cdot & \cdot \\
1 & \cdot & \cdot \\
1 & \cdot & \cdot \\
1 & \cdot & \cdot \\
1 & . & \cdot \\
\cdot & 1 & 1 \\
. & . & 1
\end{array}\right) .
$$

The first matrix leads to $k_{0}(B)=4$ and $k(B)=5$. This contradicts [17, Theorem 8.1]. The second matrix reveals $k_{0}(B)=k(B)=8$. Since Brauer's height-zero conjecture holds for $B$ by [17, Theorem 13.6], $D$ is abelian. By [17, Theorem 8.3], $D$ is not isomorphic to $C_{4} \times C_{4}$. In fact, $D$ must be elementary abelian by [18, Proposition 16], for instance. By Eaton's classification [3], $B$ should be Morita equivalent to the group algebra of the Frobenius group $D \rtimes C_{3}$. But this is a basic algebra of dimension 48 .

The local structure of $B$ is determined by a fusion system $\mathcal{F}$ on $D$ (again there are only finitely many choices for $\mathcal{F}$ when $\operatorname{dim} A$ is fixed). The $p^{\prime}$-group $E:=\operatorname{Out}_{\mathcal{F}}(D)$ is called the inertial quotient of $B$. Recall that for every $S \leq D$ there is exactly one $\operatorname{subpair}\left(S, b_{S}\right)$ attached to $\mathcal{F}$ (here, $b_{S}$ is a Brauer correspondent of $B$ in $\mathrm{C}_{G}(S)$ ). After $\mathcal{F}$-conjugation, we may and will always assume that $S$ is fully $\mathcal{F}$-normalised. Then $b_{S}$ has defect group $\mathrm{C}_{D}(S)$ and fusion system $\mathrm{C}_{\mathcal{F}}(S)$. Moreover, the Brauer correspondent $B_{S}:=b_{S}^{\mathrm{N}_{G}\left(S, b_{S}\right)}$ has defect group $\mathrm{N}_{D}(S)$ and fusion system $\mathrm{N}_{\mathcal{F}}(S)$. If $S=\langle u\rangle$ is cyclic, we call $\left(u, b_{u}\right):=\left(S, b_{S}\right)$ a subsection.

Let $\mathcal{R}$ be a set of representatives of the $\mathcal{F}$-conjugacy classes of elements in $D$. Then a formula of Brauer asserts that

$$
k(B)=\sum_{u \in \mathcal{R}} l\left(b_{u}\right)
$$

Each $b_{u}$ dominates a block $\overline{b_{u}}$ of $\mathrm{C}_{G}(u) /\langle u\rangle$ with defect group $\mathrm{C}_{D}(u) /\langle u\rangle$ and fusion system $\mathrm{C}_{\mathcal{F}}(u) /\langle u\rangle$. If $\overline{C_{u}}$ is the Cartan matrix of $\overline{b_{u}}$, then $C_{u}:=|\langle u\rangle| \overline{C_{u}}$ is the Cartan matrix of $b_{u}$. Let $Q_{u}:=\left(d_{\chi \phi}^{u}: \chi \in \operatorname{Irr}(B), \phi \in \operatorname{IBr}\left(b_{u}\right)\right)$ be the generalised decomposition matrix with respect to $\left(u, b_{u}\right)$. The orthogonality relations assert that $Q_{u}^{\mathrm{t}} \overline{Q_{v}}=\delta_{u v} C_{u}$ for $u, v \in \mathcal{R}$ where $\delta_{u v}$ is the Kronecker delta and $\overline{Q_{v}}$ is the complex conjugate of $Q_{v}$. As above, we define the contribution matrices $M^{u}$ for each $u \in \mathcal{R}$. Since the generalised decomposition numbers are algebraic integers, we may express $Q_{u}$ with respect to a suitable integral basis. This yields 'fake' decomposition matrices $\tilde{Q}_{u}$ which obey similar orthogonality relations (see [1, Section 4] for details). We call $\tilde{C}_{u}:=\tilde{Q}_{u}^{\mathrm{t}} \tilde{Q}_{u}$ the 'fake' Cartan matrix of $b_{u}$.

The following curious result might be of independent interest. 
PROpOSITION 2.3. Let $B$ be a p-block of a finite group with abelian defect group $D$ and inertial quotient $E$.

(i) If $p=2$, then $l(B) \equiv|E| \equiv k(E)(\bmod 8)$.

(ii) If $p=3$, then $l(B) \equiv|E| \equiv k(E)(\bmod 3)$.

Proof. We argue by induction on $|D|$. If $|D| \leq 4$, then $l(B)=|E|=k(E)$. Thus, let $|D| \geq 8$. Let $d:=8$ if $p=2$ and $d:=3$ if $p=3$. Let $\mathcal{R}$ be a set of representatives of the $E$-orbits on $D$. Since $E$ is a $p^{\prime}$-group, we have $\left|\mathrm{C}_{E}(u)\right|^{2} \equiv 1(\bmod d)$ for all $u \in D$. Hence,

$$
|E| \sum_{u \in \mathcal{R}}\left|\mathrm{C}_{E}(u)\right|=\sum_{u \in D}\left|\mathrm{C}_{E}(u)\right|^{2} \equiv|D| \equiv 0(\bmod d) .
$$

By Kessar and Malle [10] and [17, Proposition 1.31], $k(B)=k_{0}(B) \equiv 0(\bmod d)$. Using Brauer's formula and induction yields

$$
l(B)=k(B)-\sum_{u \in \mathcal{R} \backslash\{1\}} l\left(b_{u}\right) \equiv-\sum_{u \in \mathcal{R} \backslash\{1\}}\left|\mathrm{C}_{E}(u)\right| \equiv|E| \equiv \sum_{\chi \in \operatorname{Irr}(E)} \chi(1)^{2} \equiv k(E)(\bmod d) .
$$

For the principal block $B$, Alperin's weight conjecture asserts that $l(B)=k(E)$ in the situation of Proposition 2.3.

Finally, we study the elementary divisors of $C$ via the theory of lower defect groups. The 1-multiplicity $m_{B}^{(1)}(S)$ of a subgroup $S \leq D$ is defined as the dimension of a certain section of $\mathrm{Z}(B)$ (the precise definition in [17, Section 1.8] is not needed here). Since we are only interested in 1-multiplicities, we omit the exponent (1) from now on. Furthermore, it is desirable to attach a multiplicity to a subpair $\left(S, b_{S}\right)$ instead of a subgroup. We do so by setting

$$
m_{B}\left(S, b_{S}\right):=m_{B_{S}}(S)
$$

Note that $\left(S, b_{S}\right)$ is also a subpair for $B_{S}$ and $m_{B_{S}}\left(S, b_{S}\right)=m_{B}\left(S, b_{S}\right)$. Now the multiplicity of an elementary divisor $d$ of $C$ is

$$
m(d)=\sum m_{B}\left(S, b_{S}\right)
$$

where $\left(S, b_{S}\right)$ runs through the $\mathcal{F}$-conjugacy classes of subpairs with $|S|=d$. In particular, $m_{B}\left(D, b_{D}\right)=m(|D|)=1$.

We are now in a position to investigate blocks with extraspecial defect group $D \cong 3_{+}^{1+2}$ of order 27 and exponent 3 . The partial results on these blocks obtained by Hendren [8] are not sufficient for our purpose. We proceed in four stages. The first lemma is analogous to [17, Lemma 13.3].

LEMMA 2.4. Let $B$ be a block of a finite group $G$ with defect group $D \cong C_{3} \times C_{3}$ and inertial quotient $E \cong C_{2} \times C_{2}$. Suppose that $l(B)=4$. Let $D=S \times T$ with E-invariant subgroups $S \cong T \cong C_{3}$. Then $m_{B}\left(S, b_{S}\right)=m_{B}\left(T, b_{T}\right)=1$. 
Proof. By [1, Theorem 3], $B$ is perfectly isometric to its Brauer correspondent in $\mathrm{N}_{G}(D)$. It follows that the elementary divisors of the Cartan matrix of $B$ are $1,3,3,9$. In particular, $m(3)=2$. Let $U \leq D$ be of order 3 such that $S \neq U \neq T$. Then $b_{U}$ is nilpotent and $l\left(b_{U}\right)=1$. Since $B_{U}$ has defect group $D$, we obtain $m_{B_{U}}(D)=1$. Hence, [17, Lemma 1.43] implies $m_{B}\left(U, b_{U}\right)=m_{B_{U}}(U)=0$. It follows that

$$
m_{B}\left(S, b_{S}\right)+m_{B}\left(T, b_{T}\right)=m(3)=2 .
$$

Similarly, $b_{S}$ has defect group $D$ and inertial quotient $C_{2}$. Hence, $l\left(b_{S}\right)=2$ by [1, Theorem 3]. This time [17, Lemma 1.43] gives

$$
m_{B}\left(S, b_{S}\right)=m_{B_{S}}(S)+m_{B_{S}}(D)-1 \leq l\left(b_{S}\right)-1=1,
$$

and similarly $m_{B}\left(T, b_{T}\right) \leq 1$. By (2.1), we must have equality.

We recall that every $3^{\prime}$-automorphism group $E$ of $D \cong 3_{+}^{1+2}$ acts faithfully on $D / \Phi(D) \cong C_{3} \times C_{3}$. This allows us to regard $E$ as a subgroup of the semilinear group $\Gamma \mathrm{L}(1,9) \leq \mathrm{GL}(2,3)$. Note that $\Gamma \mathrm{L}(1,9)$ is isomorphic to the semidihedral group $S D_{16}$. Moreover, $\mathrm{C}_{E}(\mathrm{Z}(D))=E \cap \mathrm{SL}(2,3) \leq Q_{8}$.

LEMMA 2.5. Let $B$ be a block of a finite group $G$ with defect group $D \cong 3_{+}^{1+2}$ and inertial quotient $E \cong S D_{16}$. Suppose that $Z:=\mathrm{Z}(D) \unlhd G$ and that $\operatorname{IBr}\left(b_{Z}\right)$ contains at least four Brauer characters which are not $G$-invariant. Then $m_{B}\left(Z, b_{Z}\right)>0$.

Proof. Since $\mathrm{C}_{E}(Z) \cong Q_{8}$ acts regularly on $D / Z$, there are two subgroups, say $Z$ and $S$, of order 3 in $D$ up to $\mathcal{F}$-conjugation. Hence, $m(3)=m_{B}\left(Z, b_{Z}\right)+m_{B}\left(S, b_{S}\right)$. We observe that $B_{S}$ has defect group $\mathrm{N}_{D}(S)=S Z \cong C_{3} \times C_{3}$ and inertial quotient $C_{2} \times C_{2}$. By [1, Theorem 3], $l\left(B_{S}\right) \in\{1,4\}$. In the first case $m_{B}\left(S, b_{S}\right)=0$ by [17, Lemma 1.43], and in the second case $m_{B}\left(S, b_{S}\right)=m_{B_{S}}\left(S, b_{S}\right)=1$ by Lemma 2.4. Thus, it suffices to show that $m(3) \geq 2$.

Since $E$ acts nontrivially on $Z$, we have $|G: N|=2$ where $N:=\mathrm{C}_{G}(Z)$. As usual, $b_{Z}$ dominates a block $\overline{b_{Z}}$ with defect group $D / Z \cong C_{3} \times C_{3}$ and inertial quotient $\mathrm{C}_{E}(Z) \cong$ $Q_{8}$. By hypothesis, $l\left(b_{Z}\right) \geq 4$. By [1, Lemma 13], there exists a basic set $\Gamma$ for $\overline{b_{Z}}$ (which is a basic set for $b_{Z}$ as well) such that $G$ acts on $\Gamma$ and the Cartan matrix of $b_{Z}$ with respect to $\Gamma$ is

$$
3\left(\begin{array}{lllll}
2 & 1 & 1 & 1 & 2 \\
1 & 2 & 1 & 1 & 2 \\
1 & 1 & 2 & 1 & 2 \\
1 & 1 & 1 & 2 & 2 \\
2 & 2 & 2 & 2 & 5
\end{array}\right) \quad \text { or } \quad 3\left(1+\delta_{i j}\right)_{i, j=1}^{8}
$$

We may assume that $\theta_{1}, \ldots, \theta_{4} \in \Gamma$ such that $\phi:=\theta_{1}^{G}=\theta_{2}^{G}$ and $\mu:=\theta_{3}^{G}=\theta_{4}^{G}$ belong to a basic set $\Delta$ of $B$. In order to determine the Cartan matrix $C$ of $B$ with respect to $\Delta$, we introduce the projective indecomposable characters $\Phi_{\phi}$ and $\Phi_{\mu}$ (note that these are generalised characters in our setting). By [14, Theorem 8.10], $\Phi_{\phi}=\Phi_{\theta_{1}}^{G}$ and $\Phi_{\mu}=\Phi_{\theta_{3}}^{G}$. 
In particular, $\Phi_{\phi}$ and $\Phi_{\mu}$ vanish outside $N$. We compute

$$
\begin{aligned}
{\left[\Phi_{\phi}, \Phi_{\phi}\right] } & =\frac{1}{|G|} \sum_{g \in G}\left|\Phi_{\phi}(g)\right|^{2}=\frac{1}{2} \frac{1}{|N|} \sum_{g \in N}\left|\Phi_{\phi}(g)\right|^{2} \\
& =\frac{1}{2}\left[\Phi_{\theta_{1}}+\Phi_{\theta_{2}}, \Phi_{\theta_{1}}+\Phi_{\theta_{2}}\right]=9=\left[\Phi_{\mu}, \Phi_{\mu}\right], \\
{\left[\Phi_{\phi}, \Phi_{\mu}\right] } & =\frac{1}{2}\left[\Phi_{\theta_{1}}+\Phi_{\theta_{2}}, \Phi_{\theta_{3}}+\Phi_{\theta_{4}}\right]=6 .
\end{aligned}
$$

Let $\tau \in \Delta \backslash\{\phi, \mu\}$. If $\tau_{N}$ is the sum of two characters in $\Gamma$, then $l\left(b_{Z}\right)=8$ and

$$
\left[\Phi_{\phi}, \Phi_{\tau}\right]=6=\left[\Phi_{\mu}, \Phi_{\tau}\right] .
$$

If, on the other hand, $\tau_{N} \in \Gamma$, then also $\left(\Phi_{\tau}\right)_{N}=\Phi_{\tau_{N}}$ by [14, Corollary 8.8] and

$$
\left[\Phi_{\phi}, \Phi_{\tau}\right]=\left[\Phi_{\mu}, \Phi_{\tau}\right] \in\{3,6\},
$$

depending on $l\left(b_{Z}\right)$. In any case, $C$ has the form

$$
C=\left(\begin{array}{ccccc}
9 & 6 & a_{1} & \cdots & a_{s} \\
6 & 9 & a_{1} & \cdots & a_{s} \\
a_{1} & a_{1} & * & \cdots & * \\
\vdots & \vdots & \vdots & & \vdots \\
a_{s} & a_{s} & * & \cdots & *
\end{array}\right)
$$

with $a_{1}, \ldots, a_{s} \in\{3,6\}$. By the Gauss algorithm there exist $X, Y \in \operatorname{GL}(l(B), \mathbb{Z})$ such that

$$
X C Y=\left(\begin{array}{ccc}
3 & \cdot & \cdot \\
\cdot & 3 & \cdot \\
\cdot & \cdot & *
\end{array}\right) .
$$

Since all elementary divisors of $C$ are powers of 3 , it follows that $m(3) \geq 2$ as desired.

LEMMA 2.6. Let $B$ be a block of a finite group $G$ with defect group $D \cong 3_{+}^{1+2}$ and fusion system $\mathcal{F}=\mathcal{F}\left(J_{4}\right)$. Then $B$ cannot have Cartan matrix $\left(\begin{array}{ll}7 & 1 \\ 1 & 4\end{array}\right)$.

PROOF. By way of contradiction, suppose that $B$ has the given Cartan matrix $C$. Then $B$ has decomposition matrix

$$
\left(\begin{array}{cc}
2 & \cdot \\
1 & \cdot \\
1 & \cdot \\
\cdot & 1 \\
\cdot & 1 \\
\cdot & 1 \\
1 & 1
\end{array}\right) \text { or }\left(\begin{array}{cc}
1 & \cdot \\
1 & \cdot \\
1 & \cdot \\
1 & \cdot \\
1 & \cdot \\
1 & \cdot \\
\cdot & 1 \\
\cdot & 1 \\
\cdot & 1 \\
1 & 1
\end{array}\right)
$$


The diagonal of the contribution matrix $M^{1}$ is

$$
(16,4,4,7,7,7,9) \text { or }(4,4,4,4,4,4,7,7,7,9) \text {. }
$$

It follows that $k_{0}(B) \in\{6,9\}$ and $k_{1}(B)=1$ (the last row corresponds to the character of height 1). From the Atlas, all nontrivial elements of $D$ are $\mathcal{F}$-conjugate. Let $\left(z, b_{z}\right)$ be a nontrivial subsection such that $z \in Z:=\mathrm{Z}(D)$. By [16, Table 1.2], $B$ has inertial quotient $S D_{16}$. It follows that $b_{z}$ is a block with defect group $D$ and inertial quotient $Q_{8}$. Moreover, $l\left(b_{z}\right)=k(B)-l(B) \in\{5,8\}$. The possible Cartan matrices of $b_{z}$ are given in the proof of Lemma 2.5. The generalised decomposition numbers $d_{\chi \phi}^{z}$ are Eisenstein integers and can be expressed with respect to the integral basis $1, e^{2 \pi i / 3}$. According to the action of $\mathrm{N}_{G}\left(Z, b_{z}\right)$ on $\operatorname{IBr}\left(b_{z}\right)$ there are eight possibilities for the 'fake' Cartan matrix $\tilde{C}_{z}$ which are listed explicitly in [1, proof of Lemma 14]. In each case we apply an algorithm of Plesken [15] (implemented in GAP [7]) to determine the feasible 'fake' decomposition matrices $\tilde{Q}_{z}$. To this end we also take into account that the diagonal of $M^{z}$ is $(11,23,23,20,20,20,18)$ or $(23,23,23,23,23,23,20,20,20,18)$, since $M^{1}+M^{z}=|D| 1_{k(B)}$. It turns out that only two of the eight cases can actually occur. If $k(B)=7$ then $\mathrm{N}_{G}\left(Z, b_{z}\right)$ has one fixed point in $\operatorname{IBr}\left(b_{z}\right)$, and if $k(B)=10$ then $\mathrm{N}_{G}\left(Z, b_{z}\right)$ has two fixed points in $\operatorname{IBr}\left(b_{z}\right)$. Hence, in both cases the block $B_{Z}$ fulfils the assumption of Lemma 2.5. Consequently, $m(3)=m_{B}\left(Z, b_{Z}\right)=m_{B_{Z}}\left(Z, b_{Z}\right)>0$. However, the elementary divisors of $C$ are 1 and 27, a contradiction.

PROPOSITION 2.7. There does not exist a block of a finite group with Cartan matrix $\left(\begin{array}{ll}7 & 1 \\ 1 & 4\end{array}\right)$.

Proof. As in Lemma 2.6, any block $B$ with the given Cartan matrix $C$ has a defect group $D$ of order 27 . The possible decomposition matrices were also computed in the proof of Lemma 2.6. In particular, $k_{0}(B) \in\{6,9\}, k_{1}(B)=1$ and $k(B)-l(B) \in\{5,8\}$. By Kessar and Malle [10], $D$ is nonabelian. By [17, Theorem 8.15], $D$ cannot have exponent 9 , that is, $D \cong 3_{+}^{1+2}$. The fusion systems $\mathcal{F}$ on that group were classified in Ruiz and Viruel [16]. As explained before, we regard the inertial quotient $E$ of $B$ as a subgroup of $S D_{16}$. Let $\mathcal{R}$ be a set of representatives of the $\mathcal{F}$-conjugacy classes in $D$. For $1 \neq u \in \mathcal{R}$, we have $l\left(b_{u}\right) \equiv\left|\mathrm{C}_{E}(u)\right|(\bmod 3)$ by Proposition 2.3 (applied to $\overline{b_{u}}$ if $u \in Z:=\mathrm{Z}(D))$. Therefore, the residue of $k(B)-l(B)$ modulo 3 only depends on $\mathcal{F}$. If $D$ contains $\mathcal{F}$-essential subgroups, then $\mathcal{F}$ is the fusion system of one of the following groups $H$ :

$$
C_{3}^{2} \rtimes \operatorname{SL}(2,3), C_{3}^{2} \rtimes \operatorname{GL}(2,3), \operatorname{PSL}(3,3), \operatorname{PSL}(3,3) .2,{ }^{2} F_{4}(2)^{\prime}, J_{4} .
$$

The last case was excluded in Lemma 2.6. In the remaining cases we can compare with the principal block of $H$ to derive the contradiction

$$
2 \equiv k(B)-l(B) \equiv k\left(B_{0}(H)\right)-l\left(B_{0}(H)\right) \not \equiv 2(\bmod 3) .
$$

Hence, there are no $\mathcal{F}$-essential subgroups, that is, $\mathcal{F}=\mathcal{F}(D \rtimes E)$. Suppose that $E \leq Q_{8}$. Then $\mathrm{N}_{G}\left(Z, b_{Z}\right)=\mathrm{C}_{G}(Z)$ and $b_{Z}=B_{Z}$ has fusion system $\mathcal{F}$ as well. If $E=1$, then $B$ is nilpotent in contradiction to $l(B)=2$. Thus, let $E \neq 1$. Let $\overline{B_{Z}}$ be the block 
with defect group $D / Z$ dominated by $B_{Z}$. By [1, Theorem 3] and Proposition 2.3, $l\left(B_{Z}\right)=l\left(\overline{B_{Z}}\right) \geq 2$. Since $E$ acts semiregularly on $D / Z$, the Cartan matrix of $\overline{B_{Z}}$ has elementary divisors 1 and 9 (see [17, Proposition 1.46]). Hence, 3 is an elementary divisor of the Cartan matrix of $B_{Z}$. Since $Z \leq Z\left(C_{G}(Z)\right)$, it follows that $m(3) \geq$ $m_{B}\left(Z, b_{Z}\right)=m_{B_{Z}}\left(Z, b_{Z}\right)>0$ by [17, Lemma 1.44], a contradiction.

We are left with the situation $E \nsubseteq Q_{8}$. Here, $\mathcal{R} \cap Z=\{1, z\}$. The case $E \cong C_{2} \times C_{2}$ is impossible by a comparison with the principal block of $D \rtimes E$ as above. We summarise the remaining cases (the second column refers to the small groups library in GAP).

\begin{tabular}{cccc}
$E$ & realising group & $l\left(b_{z}\right)$ & $\sum_{u \notin \mathrm{Z}(D)} l\left(b_{u}\right)$ \\
\hline$C_{2}$ & $54: 5$ & 1 & $2+2+1+1+1$ \\
$C_{8}$ & $216: 86$ & 4 & 1 \\
$D_{8}$ & $216: 87$ & 4 & $2+2$
\end{tabular}

In the first case, $k_{0}(B)=9$ and there exists $u \in \mathcal{R} \backslash Z$ such that $u$ and $u^{-1}$ are $\mathcal{F}$-conjugate. Then $l\left(b_{u}\right)=1$ and the Cartan matrix of $b_{u}$ is (9). The generalised decomposition matrix $Q_{u}$ is integral, since $Q_{u}=Q_{u^{-1}}=\overline{Q_{u}}$. The only choice up to signs is $Q_{u}=( \pm 1, \ldots, \pm 1,0)^{\mathrm{t}}$ where the last character has height 1 . However, $Q_{u}$ cannot be orthogonal to the decomposition matrix of $B$ as computed in Lemma 2.6. Next let $E \cong C_{8}$. Here $k_{0}(B)=6$ and the generalised decomposition matrix $Q_{u}$ has the form $Q_{u}=( \pm 2, \pm 1, \ldots, \pm 1,0)^{\mathrm{t}}$. More precisely, $Q_{1}$ and $Q_{u}$ can be arranged as

$$
\left(Q_{1}, Q_{u}\right)=\left(\begin{array}{ccc}
2 & . & 1 \\
1 & . & -1 \\
1 & . & -1 \\
\cdot & 1 & 2 \\
\cdot & 1 & -1 \\
\cdot & 1 & -1 \\
1 & 1 & .
\end{array}\right) .
$$

So we compute the diagonal of the contribution matrix $M^{z}$ as $(8,20,20,8,17,17,18)$. By [1, Proposition 7] (applied to the dominated block with defect group $C_{3} \times C_{3}$ ), there exists a basic set $\Gamma$ for $b_{z}$ such that the Cartan matrix becomes $3\left(2+\delta_{i j}\right)_{i, j=1}^{4}$ and $\mathrm{N}_{G}\left(Z, b_{Z}\right)$ acts on $\Gamma$. There are three such actions. In each case we may compute the 'fake' Cartan matrix $\tilde{C}_{z}$ and apply Plesken's algorithm. It turns out that none of those cases leads to a valid configuration.

Finally, let $E \cong D_{8}$ and $u \in \mathcal{R}$ such that $l\left(b_{u}\right)=2$. We check that $u$ is $\mathcal{F}$-conjugate to $u^{-1}$. The Cartan matrix of $b_{u}$ is $3\left(\begin{array}{ll}2 & 1 \\ 1 & 2\end{array}\right)$ up to basic sets. Let $U:=\langle u\rangle$. If $\mathrm{N}_{G}\left(U, b_{u}\right)$ interchanges the Brauer characters of $b_{u}$, then the 'fake' Cartan matrix becomes $\tilde{C}_{u}=\left(\begin{array}{ll}5 & 1 \\ 1 & 2\end{array}\right)$ (see [1, proof of Lemma 14]). But then $k_{0}(B) \leq 6$, which is not the case. Therefore, $\mathrm{N}_{G}\left(U, b_{u}\right)$ fixes the Brauer characters of $b_{u}$ and $B_{U}$ satisfies $l\left(B_{U}\right)=4$ by Clifford theory. By Lemma 2.4, we conclude that $m(3) \geq m_{B}\left(U, b_{U}\right)=m_{B_{U}}\left(U, b_{U}\right)=1$. This is the final contradiction. 
Since the contribution matrix does not depend on basic sets, the proof shows more generally that $\left(\begin{array}{ll}7 & 1 \\ 1 & 4\end{array}\right)$ cannot be the Cartan matrix of a block with respect to any basic set. This is in contrast to the main result of [12] where the authors showed that $\left(\begin{array}{ll}5 & 1 \\ 1 & 2\end{array}\right)$ is not the Cartan matrix of a block with defect group $C_{3} \times C_{3}$, although a transformation of basic sets results in the Cartan matrix

$$
\left(\begin{array}{ll}
5 & 4 \\
4 & 5
\end{array}\right)=\left(\begin{array}{cc}
1 & 0 \\
1 & -1
\end{array}\right)\left(\begin{array}{cc}
5 & 1 \\
1 & 2
\end{array}\right)\left(\begin{array}{cc}
1 & 1 \\
0 & -1
\end{array}\right)
$$

of the Frobenius group $C_{3}^{2} \rtimes C_{2}$.

\section{Basic algebras of dimension 13}

Suppose that $B$ is a block with basic algebra $A$ of dimension 13 and Cartan matrix $C$. We discuss the various possibilities for $C$. If $l(B)=1$ then $C=(13), p=13$ and $B$ has defect 1 . This is covered by Proposition 2.1. For $l(B)=2$ we obtain the following possibilities for $C$ up to labelling of the simple modules:

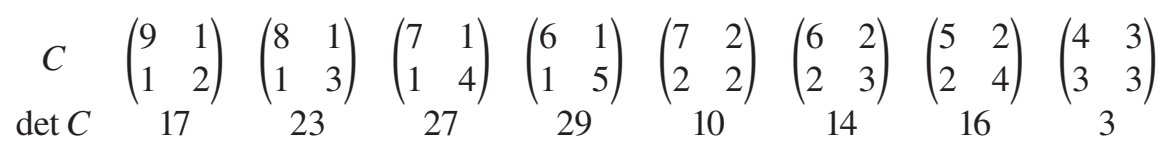

The determinants 10 and 14 are not prime powers. If $\operatorname{det} C$ is a prime, then the result follows from Proposition 2.1. The remaining cases with $\operatorname{det} C \in\{16,27\}$ were handled in Proposition 2.2 and Proposition 2.7, respectively.

We now turn to $l(B)=3$. Up to labelling, the following possibilities may arise:

$$
\begin{aligned}
& C \quad\left(\begin{array}{lll}
5 & 1 & 1 \\
1 & 2 & 0 \\
1 & 0 & 2
\end{array}\right)\left(\begin{array}{lll}
5 & 1 & 0 \\
1 & 2 & 1 \\
0 & 1 & 2
\end{array}\right)\left(\begin{array}{lll}
4 & 1 & 1 \\
1 & 3 & 0 \\
1 & 0 & 2
\end{array}\right)\left(\begin{array}{lll}
4 & 1 & 0 \\
1 & 3 & 1 \\
0 & 1 & 2
\end{array}\right)\left(\begin{array}{lll}
4 & 0 & 1 \\
0 & 3 & 1 \\
1 & 1 & 2
\end{array}\right) \\
& \begin{array}{llllll}
\operatorname{det} C & 16 & 13 & 19 & 18 & 17
\end{array} \\
& \begin{array}{r}
C \\
\operatorname{det} C
\end{array}\left(\begin{array}{lll}
3 & 1 & 1 \\
1 & 3 & 0 \\
1 & 0 & 3
\end{array}\right)\left(\begin{array}{lll}
3 & 1 & 1 \\
1 & 2 & 1 \\
1 & 1 & 2
\end{array}\right)\left(\begin{array}{lll}
3 & 2 & 0 \\
2 & 2 & 1 \\
0 & 1 & 2
\end{array}\right)\left(\begin{array}{lll}
3 & 2 & 1 \\
2 & 2 & 0 \\
1 & 0 & 2
\end{array}\right)
\end{aligned}
$$

The determinants 1, 18 and 21 are impossible and the prime determinants are settled by Proposition 2.1. The remaining case was dealt with in Proposition 2.2.

If $l(B) \geq 4$, then the trace of $C$ is greater than or equal to 8 . Since $C$ is symmetric and indecomposable, we need at least six more nonzero entries. But then $\operatorname{dim} A \geq$ $8+6=14$.

\section{Basic algebras of dimension 14}

In this section, $B$ is a block with basic algebra $A$ of dimension 14 . Since 14 is not a prime power, $l(B) \geq 2$. In view of Proposition 2.1, we only list the possible Cartan matrices $C$ such that $\operatorname{det} C$ is a prime power, but not a prime: 


$$
\begin{aligned}
& C \quad\left(\begin{array}{lll}
6 & 1 & 0 \\
1 & 2 & 1 \\
0 & 1 & 2
\end{array}\right)\left(\begin{array}{lll}
5 & 1 & 1 \\
1 & 3 & 0 \\
1 & 0 & 2
\end{array}\right)\left(\begin{array}{lll}
4 & 2 & 0 \\
2 & 2 & 1 \\
0 & 1 & 2
\end{array}\right)\left(\begin{array}{lll}
3 & 1 & 0 \\
1 & 3 & 2 \\
0 & 2 & 2
\end{array}\right)\left(\begin{array}{llll}
2 & 1 & 1 & 1 \\
1 & 2 & 0 & 0 \\
1 & 0 & 2 & 0 \\
1 & 0 & 0 & 2
\end{array}\right) \\
& \begin{array}{llllll}
\operatorname{det} C & 16 & 25 & 4 & 4 & 4
\end{array}
\end{aligned}
$$

The 2-blocks of defect 2 were classified by Erdmann [5]. The Morita equivalence classes are represented by $F D, F A_{4}$ and $B_{0}\left(A_{5}\right)$. Only the last block did not already appear in Linckelmann's list. It is easy to check that $B_{0}\left(A_{5}\right)$ has a basic algebra of dimension 18. The case $\operatorname{det} C=16$ was dealt with in Proposition 2.2. Now let $\operatorname{det} C=$ 25 and $p=5$. Since $l(B)=3$ does not divide $p-1=4, D$ is elementary abelian of order 25 . The decomposition matrix is

$$
\left(\begin{array}{lll}
1 & 0 & 0 \\
1 & 0 & 0 \\
1 & 0 & 0 \\
1 & 1 & 0 \\
1 & 0 & 1 \\
0 & 1 & 0 \\
0 & 1 & 0 \\
0 & 0 & 1
\end{array}\right) .
$$

In particular, $k(B)-l(B)=5$. Let $E \leq \mathrm{GL}(2,5)$ be the inertial quotient of $B$. Every nontrivial subsection $\left(u, b_{u}\right)$ satisfies $l\left(b_{u}\right)=\left|\mathrm{C}_{E}(u)\right|$ by Brauer-Dade theory. In particular, $k(B)-l(B)$ only depends on the action of $E$ on $D$. An inspection of [1, Theorem 5] shows that $k(B)-l(B)=5$ never occurs. Hence, this case is impossible as well.

\section{The next challenge}

While classifying blocks $B$ with basic algebra of dimension 15 , only the following Cartan matrices are hard to deal with:

$$
\left(\begin{array}{lll}
5 & 1 & 1 \\
1 & 2 & 1 \\
1 & 1 & 2
\end{array}\right), \quad\left(\begin{array}{lll}
6 & 0 & 1 \\
0 & 3 & 1 \\
1 & 1 & 2
\end{array}\right) .
$$

The first matrix belongs to a Brauer tree algebra and could potentially arise from a 13-block of defect 1 (see Proposition 2.1). David Craven has informed me that such a block most likely does not exist (assuming the classification of finite simple groups).

The second matrix leads, once again, to a defect group $D$ of order 27. Moreover, $k(B)=k_{0}(B) \in\{6,9\}$. Arguing along the lines of Proposition 2.7, it can be shown with some effort that $D$ is abelian. Now the block is ruled out by Proposition 2.3.

Finally, for basic algebras of dimension 16, a $3 \times 3$ Cartan matrix with largest elementary divisor 32 shows up. We made no attempt to say something about such blocks. 


\section{Acknowledgement}

I thank David Craven for providing detailed information on the possible trees of block algebras.

\section{References}

[1] C. G. Ardito and B. Sambale, 'Cartan matrices and Brauer's $k(B)$-Conjecture V', Preprint, https://arxiv.org/abs/1911.10710v1.

[2] C. Bonnafé, Representations of $S L_{2}\left(F_{q}\right)$, Algebra and Applications, 13 (Springer-Verlag, London, UK, 2011).

[3] C. Eaton, 'Morita equivalence classes of blocks with elementary abelian defect groups of order 16', Preprint, https://arxiv.org/abs/1612.03485v4.

[4] C. W. Eaton and M. Livesey, 'Donovan's conjecture and blocks with abelian defect groups', Proc. Amer. Math. Soc. 147 (2019), 963-970.

[5] K. Erdmann, Blocks of Tame Representation Type and Related Algebras, Lecture Notes in Mathematics, 1428 (Springer-Verlag, Berlin, 1990).

[6] P. Fong and B. Srinivasan, 'Brauer trees in GL (n, q)', Math. Z. 187 (1984), 81-88.

[7] The GAP Group, GAP - Groups, Algorithms, and Programming, Version 4.11.0 (2020), http://www.gap-system.org.

[8] S. Hendren, 'Extra special defect groups of order $p^{3}$ and exponent p', J. Algebra 313 (2007), 724-760.

[9] T. Holm, Blocks of Tame Representation Type and Related Algebras: Derived Equivalences and Hochschild Cohomology, Habilitationsschrift, Magdeburg, 2001.

[10] R. Kessar and G. Malle, 'Quasi-isolated blocks and Brauer's height zero conjecture', Ann. Math. (2) 178 (2013), 321-384.

[11] M. Linckelmann, 'Finite-dimensional algebras arising as blocks of finite group algebras', in: Representations of Algebras, Contemporary Mathematics, 705 (American Mathematical Society, Providence, RI, 2018), 155-188.

[12] M. Linckelmann and W. Murphy, 'A 9-dimensional algebra which is not a block of a finite group', Preprint, https://arxiv.org/abs/2005.02223v1.

[13] N. Naehrig, 'A construction of almost all Brauer trees', J. Group Theory 11 (2008), 813-829.

[14] G. Navarro, Characters and Blocks of Finite Groups, London Mathematical Society Lecture Note Series, 250 (Cambridge University Press, Cambridge, UK, 1998).

[15] W. Plesken, 'Solving $X X^{\mathrm{tr}}=A$ over the integers', Linear Algebra Appl. 226/228 (1995), 331-344.

[16] A. Ruiz and A. Viruel, 'The classification of $p$-local finite groups over the extraspecial group of order $p^{3}$ and exponent p', Math. Z. 248 (2004), 45-65.

[17] B. Sambale, Blocks of Finite Groups and their Invariants, Springer Lecture Notes in Mathematics, 2127 (Springer-Verlag, Cham, 2014).

[18] B. Sambale, 'Cartan matrices and Brauer's k(B)-Conjecture IV', J. Math. Soc. Jpn. 69 (2017), 1-20.

[19] R. A. Wilson, J. G. Thackray, R. A. Parker, F. Noeske, J. Müller, K. Lux, F. Lübeck, C. Jansen, G. Hiss and T. Breuer, The Modular Atlas homepage, http://www.math.rwth-aachen. de/ MOC/decomposition/tex/A7/A7mod7.pdf .

BENJAMIN SAMBALE,

Institut für Algebra, Zahlentheorie und Diskrete Mathematik, Leibniz Universität Hannover, Welfengarten 1, 30167 Hannover, Germany

e-mail: sambale@math.uni-hannover.de 